\title{
Diseñando organizaciones verdes. Análisis de la relación entre el diseño organizativo y las prácticas avanzadas de gestión ambiental
}

\author{
Miguel Pérez Valls \\ José Céspedes Lorente \\ Raquel Antolín López \\ Universidad de Almería
}

\section{Resumen}

La implementación de prácticas avanzadas de gestión ambiental es un proceso complejo que requiere de coordinación entre las distintas unidades funcionales de la organización y de cambios significativos en los procesos vinculados al desarrollo de las operaciones. La estructura organizativa juega por tanto un papel clave en su desarrollo. En este trabajo analizaremos la relación existente entre variables organizativas en términos de la complejidad, formalización y centralización de la estructura y las prácticas ambientales. Para contrastar estas relaciones se empleará una muestra de 109 empresas europeas dedicadas al transporte de pasajeros por vía aérea. Los resultados obtenidos del análisis sugieren que un mayor grado de descentralización, formalización de las tareas rutinarias y horizontalidad inciden positivamente en el desarrollo de prácticas avanzadas de gestión ambiental.

Palabras clave: estructura organizativa, prácticas de gestión ambiental, teoría de recursos y capacidades.

Clasificación JEL: L22, M19, Q56.

\begin{abstract}
The implementation of advanced environmental management practices is a complex process that requires cross-functional coordination and substantial operational changes. Thus organizational structure plays a central role in the generation of these practices. In this paper we analyze the relationship between different organizational variables (i.e. formalization, centralization and complexity) and environmental management practices. In order to test the hypotheses proposed we have used a sample of 109 firms coming from the European air passenger transport. Results suggest that a higher degree of decentralization, formalization of non-rutinary tasks and horizontality are positively related to the implementation of advanced environmental practices.
\end{abstract}

Keywords: organizational structure, environmental management practices, resource based view.

JEL classification: L22, M19, Q56.

\section{Introducción}

La gestión ambiental de la empresa, puede ser considerada como una capacidad clave para la obtención de la ventaja competitiva (Hart, 1995; Russo y Fouts, 1997; Sharma y Vredenburg, 1998) que además, bajo el enfoque de recursos y capaci- 
dades ha sido descrita como una capacidad dinámica (Aragón Correa y Sharma, 2003).

Las capacidades dinámicas permiten a las organizaciones identificar y aprovechar oportunidades a la vez que les facilitan su transformación y cambio (Teece et al., 1997). Para ello, estas capacidades deben hundir sus raíces en ciertas prácticas, procesos y estructuras de la organización (Teece, 2007). La estructura organizativa de una empresa facilita la asignación de recursos para el desarrollo de las tareas y establece la forma en la que se coordinan las actividades. La forma en que esto se lleve a cabo condicionará, por tanto, el desarrollo de las prácticas ambientales de la organización (Russo y Harrison, 2005). De esta forma, el diseño organizativo se configura como un elemento clave que define el modo en el que las estrategias se definen o forman en la empresa a la vez que posibilita su implementación. La estructura de la empresa es por tanto «el vehículo por el cual las empresas reconocen la necesidad de adaptación, determinan cómo se desarrollará ésta y hacen efectivo el cambio» (Greenwood y Miller, p.79). Parece pues interesante analizar qué características de la estructura organizativa de una empresa pueden incidir sobre el desarrollo y la implementación de estrategias ambientales proactivas.

La proactividad ambiental, entendida como la implementación voluntaria de prácticas e iniciativas que tienen por objetivo la mejora del rendimiento ambiental, puede ponerse de manifiesto a través de diferentes estrategias, cada una de ellas caracterizada por una serie de prácticas ambientales (Aragón Correa, 1998). González de Benito y González de Benito (2006) clasifican estas prácticas en tres categorías: prácticas de planificación y organizativas, prácticas operativas y prácticas de comunicación.

Las prácticas de planificación y organizativas, que reflejan el grado hasta el cual los sistemas de gestión ambiental (Enviromental Management Systems, EMS) han sido desarrollados e implementados en la organización. Estas prácticas ponen de manifiesto hasta qué punto la organización ha establecido su política ambiental y miden el impacto que tiene sobre el medio ambiente.

Las prácticas operativas implican cambios en los sistemas de producción y en las operaciones de la organización. Abarcan tanto el diseño de los productos o servicios como los procesos necesarios para su producción.

Finalmente, las prácticas de comunicación tienen por objetivo dar a conocer el impacto ambiental que tienen las operaciones de las empresas sobre el medio ambiente. Son fruto del compromiso de transparencia que caracteriza la Responsabilidad Social Empresarial (Waddock et al., 2003). Se consideran como una faceta más del compromiso ambiental de las empresas.

Las prácticas avanzadas de gestión ambiental, están íntimamente ligadas al cambio y a la proactividad. Para poder implementarlas con éxito, es necesario desarrollar estructuras organizativas que favorezcan la innovación y el cambio (Aragón Correa, 1998). Tal y como apuntan Russo y Fouts (1997: 542): «Aunque un estilo de gestión burocrático puede asociarse con un enfoque ambiental basado en el cumplimiento de la legalidad, el uso de un estilo más orgánico es necesario para capturar los bene- 
ficios asociados a comportamientos que van más allá del mero cumplimiento, dado que este tipo de estructuras generan una cultura innovadora (Aiken y Hage, 1971) que favorece los esfuerzos de prevención».

La función principal de la estructura de una organización es el reparto y distribución de los recursos y tareas necesarios para el desarrollo de su actividad, así como su posterior coordinación y control (Mintzberg, 1979). Teniendo esto en cuenta, la forma en la que una empresa desarrolle estas labores estará condicionada tanto por las características de los recursos y tareas a repartir, como por las características del entorno competitivo en el que se desenvuelve la organización. Adler, Goldoftas y Levine (1999) sugieren que las burocracias tradicionales, caracterizadas por la estandarización, especialización, formalización y jerarquía proporcionan un funcionamiento eficiente para competir en entornos estables, pero que de igual modo limitan la flexibilidad organizativa necesaria para cumplir satisfactoriamente con los requisitos de entornos complejos, ambiguos e inestables. En este sentido, desde una perspectiva integradora, Child y McGrath (2001), añaden que las burocracias tradicionales encuentran cada vez más dificultades para desempeñarse correctamente. Los motivos argumentados se fundamentan en el cambio que se está produciendo en la actualidad hacia economías intensivas en información, más que en bienes físicos. Esto hace que para poder obtener ventaja competitiva, las empresas deban generar nuevo conocimiento de una manera continuada, cuestión esta que no se puede desarrollar eficientemente con la estructura de control, totalmente reglamentada que presentan las burocracias. Sin embargo, los requerimientos del entorno hacen necesario la adopción de determinadas prácticas sustancialmente diferentes de las burocracias tradicionales (Harrison, 2005; Sánchez Bueno y Suárez González, 2010).

El objetivo del presente artículo es el de analizar la relación existente entre diversas características organizativas que se alejan de los postulados burocráticos con la implementación de prácticas avanzadas de gestión ambiental. Para ello, analizamos las dimensiones que tradicionalmente se han usado para definir estas estructuras organizativas (formalización, complejidad y centralización) y estudiamos cómo pueden influir sobre la implementación de prácticas ambientales. Al hacer esto damos un paso más hacia la concreción de las características organizativas que favorecen la implementación de un enfoque ambiental proactivo. Poner el foco sobre tres dimensiones estructurales concretas y su relación con prácticas ambientales determinadas, nos permite «eliminar capas de agregación para medir las conexiones entre las variables organizativas y determinados resultados ambientales» (Russo y Harrison, 2005: 582).

El artículo se estructura de la siguiente manera. Tras esta introducción se establece el marco teórico en el que se analizan cuáles son las dimensiones utilizadas para caracterizar tradicionalmente las formas organizativas con el fin de justificar las hipótesis propuestas posteriormente sobre su impacto en el desarrollo de prácticas avanzadas de gestión ambiental. A continuación se describe la metodología seguida en el análisis empírico, así como los resultados obtenidos. Por último se discuten dichos resultados, las implicaciones teóricas y las posibles limitaciones y extensiones del presente trabajo. 


\section{Teoría e hipótesis}

La estructura organizativa puede considerarse como un constructo multidimensional, compuesto por múltiples variables que, en función del valor que tomen, darán lugar a las diferentes configuraciones. A continuación nos centraremos en el análisis de las variables que tradicionalmente se han usado para caracterizar este constructo. De esta forma, cada estudio, en función de sus intereses incorpora, agrupa o desagrega variables como parámetros de diseño de la estructura organizativa. No obstante, existe cierto consenso en la literatura clásica acerca de que las dimensiones principales son tres: complejidad, centralización y formalización. (Child, 1974; Ford y Slocum, 1977; Hage y Aiken, 1969).

\subsection{Complejidad estructural y prácticas ambientales}

La complejidad de una estructura determinada hace referencia a los parámetros utilizados durante el proceso de diferenciación estructural, es decir del reparto del trabajo en tareas. Existen tres tipos básicos de diferenciación: horizontal, vertical y espacial.

La diferenciación horizontal hace referencia a la división del trabajo en tareas y subtareas en un mismo nivel organizativo. Algunos investigadores la equiparan al grado de especialización ocupacional, la actividad profesional específica y la formación profesional necesaria para la realización de tareas concretas (Hage y Aiken, 1969).

La diferenciación vertical incorpora la separación entre la realización del trabajo y la administración del mismo, lo que se puede concretar en el número de niveles jerárquicos. Mediante esta dimensión se han estudiado ciertas características organizativas, como el carácter vertical frente al aplanamiento de una determinada estructura y su impacto sobre la flexibilidad. (Khandwalla, 1977; Quinn, 1985; Tushman y Anderson, 1997). Estos autores establecen que las estructuras planas, frente a las altas, favorecen la adaptación a los cambios al facilitar la rápida transmisión de información desde los que la poseen a los que toman decisiones y viceversa, permitiendo, la transmisión horizontal de información además de la vertical.

Finalmente, la diferenciación espacial indica la separación en distintos puntos geográficos de las actividades y del personal.

El nuevo entorno competitivo ha puesto a las estructuras jerárquicas clásicas bajo una doble presión. En primer lugar, el elevado número de niveles de mandos intermedios supone un coste excesivo. En segundo lugar, estos niveles restringen la fluidez de la información y la rapidez de respuesta necesaria para conseguir la flexibilidad estratégica y la innovación. Como respuesta a esta situación, se ha generalizado entre las empresas procesos de reducción de niveles intermedios con la finalidad de eliminar estas caras barreras de acción (Freeman y Cameron, 1993). 
La adopción de prácticas ambientales es un proceso complejo que requiere de una coordinación interfuncional y de cambios significativos en la forma de hacer las cosas (Russo y Fouts, 1997). Las prácticas ambientales requieren del esfuerzo conjunto de todos los trabajadores y divisiones de una empresa, los cuales deben estar comprometidos con la mejora en el rendimiento ambiental (Hart, 1995). Un elevado número de niveles jerárquicos y una especialización excesiva puede dificultar la comunicación relevante para la toma de decisiones ambientales, a la vez que inhibir la participación de los miembros de la organización.

La reducción del número de niveles jerárquicos en una empresa y el desarrollo de relaciones horizontales entre distintas partes de la empresa hace que los directivos deban ser más conscientes de los procesos que guían las operaciones del día a día, a la vez que potencia que los trabajadores de base desarrollen habilidades directivas que les permitan sacar adelante su trabajo. Esto facilita el flujo de información y conocimiento en relación a las prácticas ambientales vinculadas a las operaciones tales como regulaciones y normativas o mejores prácticas (Russo y Harrison, 2005). Del mismo modo facilita que las decisiones ambientales estratégicas sean más fácilmente implementadas. De hecho, el número de miembros del staff involucrado en este tipo de actividades, está positivamente correlacionado con la adopción de prácticas pro-ambientales (Lenox y King, 2004). Algunos autores sugieren que unos flujos de comunicación vertical efectivos y adecuados, también mejoran la implementación de prácticas ambientales en la empresa (Lenox y King, 2004; Sharma et al., 1999).

Por todos estos motivos concluimos que:

H1 «Un menor número de niveles jerárquicos y una mayor intensidad en las comunicaciones horizontales están relacionadas directa y positivamente con el desarrollo de prácticas avanzadas de gestión ambiental».

\subsection{Centralización de las decisiones y prácticas ambientales}

El fenómeno centralización-descentralización se refiere a la difusión del poder de toma de decisiones. Cuando todo el poder descansa sobre un único punto de una organización, se trata de una estructura centralizada; en la medida en que el poder se dispersa entre muchos individuos, se habla de una estructura relativamente descentralizada (Mintzberg, 1979).

La eliminación de capas intermedias en la organización suele ir acompañada por una descentralización creciente, tanto a nivel operativo como estratégico. La descentralización, en áreas como «diseño de productos» y «marketing», permite mejorar los tiempos de respuesta al igual que aprovechar el conocimiento táctico de los directivos de línea (Baum y Wally, 2003). Los procesos de descentralización van acompañados habitualmente de procesos de capacitación para que los trabajadores adquieran las destrezas necesarias para desarrollar su actividad de forma correcta. Este proceso de empowerment incrementa tanto la motivación de los trabajadores 
como la implicación de los directivos, lo que puede tener un impacto positivo sobre las prácticas de gestión ambiental (Del Brío et al., 2007).

La implantación de un sistema de prácticas de gestión ambiental es un proceso intensivo en capital humano y que depende en gran medida del desarrollo de habilidades tácitas por parte de los empleados (Hart, 1995; Del Brío y Junquera, 2003). Por ello, no puede limitarse únicamente a la toma de medidas por parte del departamento responsable de cuestiones ambientales, sino que requiere de la implementación de un amplio sistema de gestión del que muchas personas se encarguen de alimentar (Boiral, 2007).

Dado que la descentralización implica el reparto de la responsabilidad en todos los niveles y unidades de la organización, este proceso de implicación interfuncional se ve favorecido. Una coordinación mejorada y el establecimiento de objetivos de forma compartida son mecanismos que pueden asegurar el flujo de información interna necesario en este proceso (Child y McGrath, 2001). Estas prácticas que favorecen la participación de los recursos humanos, ayudan a la implantación de estrategias proactivas ambientalmente dado que permiten incorporar información relevante de múltiples fuentes en la toma de decisiones ambiental (Martínez del Río et al., 2012).

Así, los procesos de descentralización favorecen la flexibilidad y el cambio. Estas estructuras permiten reducir la incertidumbre y permiten la innovación necesaria para una estrategia de proactividad ambiental (Aragón Correa, 1998).

Por todos estos motivos concluimos que:

H2 «Un mayor nivel de descentralización en las operaciones está relacionado directa y positivamente con el desarrollo de prácticas avanzadas de gestión ambiental».

\subsection{Formalización de las relaciones y prácticas ambientales}

Mediante esta variable, se recoge de forma amplia, el grado de libertad de acción del que disponen los miembros de una determinada organización. Podemos distinguir, dentro de este paraguas, los conceptos de estandarización, definida como el punto hasta el que la realización de cada una de las distintas actividades organizativas es objeto de procedimientos normalizados (Khandwalla, 1977). De igual modo, la formalización propiamente dicha hace referencia a la extensión con la que las reglas, procedimientos e instrucciones se explicitan por escrito, si bien en algunos casos pueden tener un carácter no escrito al hallarse incorporadas dentro de la cultura empresarial (Pugh et al., 1968).

El control y la evaluación juegan un papel fundamental en el desarrollo de un comportamiento ambiental avanzado (Russo y Harrison, 2005). El establecimiento de procedimientos normalizados de control y reporte puede prevenir a los trabajadores de establecer comportamientos contrarios a los objetivos de la organización (Tosi, Katz y Gómez Mejía, 1997). Del mismo modo, pueden ayudar a estructurar 
el proceso de recogida de información, haciéndolo más eficiente y operativo (Boiral, 2007).

La integración de las cuestiones ambientales en la gestión del día a día empresarial se ve facilitada gracias a la implementación de sistemas de gestión ambiental (EMS) (Russo y Fouts, 1997; Kitazawa y Sarkis, 2000; Boiral, 2002). Si bien es cierto que los EMS pueden suponer una ventaja en cuanto a la planificación y estructuración de los esfuerzos en materia ambiental, pueden darse ciertas circunstancias no planeadas que hagan que su utilidad sea limitada. Igualmente, un monitoreo constante de diversas variables ambientales supone un coste de estructura considerable. Del mismo modo, es posible que la implementación de un EMS sirva más a la legitimidad social que a la mejora en el rendimiento ambiental (Boiral, 2007).

Para prevenir estos posibles desajustes, las organizaciones pueden optar por dar una mayor libertad a sus trabajadores para establecer los cauces de actuación más adecuados. Esto se revela de especial utilidad cuando se enfrentan a tareas que se alejan de la cotidianeidad y que requieran de una mayor rapidez de respuesta (Baum y Wally, 2003) y riqueza en la solución. Una comunicación mejorada dentro de la organización puede ayudar a identificar problemas ambientales y anima a los empleados a sugerir nuevas ideas (Boiral, 2002).

Por todos estos motivos concluimos que:

H3a «Un mayor nivel de formalización en las tareas rutinarias está relacionado directa y positivamente con el desarrollo de prácticas avanzadas de gestión ambiental».

$H 3 b$ «Un menor nivel de formalización en las tareas no-rutinarias está relacionado directa y positivamente con el desarrollo de prácticas avanzadas de gestión ambiental».

\section{Desarrollo empírico}

\subsection{Muestra}

Para contrastar las cuatro hipótesis precedentes, se ha usado una muestra de empresas dedicadas al transporte de pasajeros por vía aérea en Europa. Esta elección se debe a diferentes motivos. En primer lugar, el sector de la aviación está considerado como una de las industrias más dinámicas y competitivas (Adler y Gellman, 2012), lo que favorece la emergencia de innovaciones organizativas (Galunic y Eisenhardt, 2001). En segundo lugar, esta industria presenta unas elevadas tasas de crecimiento y de cambio, requiere de inversiones sustanciales en infraestructura y para sus operaciones, se apoya fundamentalmente en el uso de combustibles fósiles. Esto hace que tanto su impacto ambiental en términos de ruido y emisiones contaminantes sea sustancial y muy visible (Hooper y Greenall, 2005). 
El universo muestral estuvo compuesto por 578 empresas provenientes de los 27 países de la Unión Europea más Noruega y Suiza. Se envió un cuestionario a dos respondentes claves de cada organización (el CEO y el jefe de operaciones). Estos individuos fueron escogidos debido a su posición en la organización y sus conocimientos sobre las cuestiones centrales de este estudio. Con el fin de incrementar la tasa de respuesta, se contactó a las empresas tanto por correo electrónico como por teléfono con el fin de confirmar la identidad del respondiente así como informarle del envío del cuestionario y de los objetivos y pretensiones del estudio. El proceso de recogida de datos se desarrolló en sucesivas fases de contacto y se llevó a cabo entre septiembre de 2009 y Junio de 2010. Finalmente se obtuvieron 109 cuestionarios válidos, lo que supone una tasa de respuesta del 18,9 por 100. Aunque baja, esta tasa de respuesta está por encima de la horquilla del 10-12 por 100 que usualmente se obtienen en los estudios dirigidos a CEOs americanos (Hambrick, Geletkanycz, y Fredrickson, 1993). Se realizó un análisis de la varianza (ANOVA) para comprobar la existencia de diferencias significativas entre las empresas que respondieron el cuestionario y las que no en función del número de empleados, activos y edad. Los resultados no mostraron diferencias significativas en ninguna de estas variables. También se compararon las respuestas de las empresas recibidas en la primera oleada con las recibidas después de los sucesivos contactos telefónicos y por email. La comparación de medias y las correlaciones de las respuestas recibidas en las diferentes oleadas no ofrecen diferencias significativas en los niveles de las variables o las relaciones entre las mismas. Los datos fueron normalizados con el fin de posibilitar una comparación homogénea de los resultados.

La base de datos fue complementada con información adicional proveniente de los informes anuales publicitados por las aerolíneas, tanto financieros como ambientales (cuando estos estaban disponibles).

\subsection{Escalas de medición utilizadas}

\subsubsection{Variables estructurales}

\section{- Complejidad}

Para medir la complejidad se han integrado diferentes variables que recogen el grado en el que la empresa reduce el número de niveles jerárquicos y la intensidad con la que se realizan conexiones horizontales entre distintas unidades o divisiones de la empresa. De esta forma se incorpora el grado en el que las organizaciones se alejan de la verticalidad y especialización que caracteriza a las burocracias tradicionales (Child y McGrath, 2001). El Cuadro 1 recoge las escalas empleadas. 


\section{CUADRO 1 \\ ESCALA COMPLEJIDAD}

\begin{tabular}{|l|l|l|l|}
\hline \multicolumn{1}{|c|}{ Variable } & \multicolumn{1}{|c|}{ Descripción } & \multicolumn{1}{c|}{ Medición } & \multicolumn{1}{c|}{ Fuente } \\
\hline $\begin{array}{l}\text { Reducción del número } \\
\text { de niveles organizativos } \\
\text { (DELAYERING) }\end{array}$ & $\begin{array}{l}\text { Evolución del número } \\
\text { de niveles organizativos } \\
\text { de la empresa en los úl- } \\
\text { timos cinco años }\end{array}$ & Escala likert 1-5 & Pettigrew et al., 2000 \\
\hline $\begin{array}{l}\text { HORIZONTAL } \\
\text { RELATIONS (HORIZ) }\end{array}$ & $\begin{array}{l}\text { Grado de unión entre } \\
\text { las subunidades/de- } \\
\text { partamentos con el fin } \\
\text { de: prestar el servicio, } \\
\text { compartir información, } \\
\text { coordinar compras con- } \\
\text { juntas, compartir infor- } \\
\text { mación comercial }\end{array}$ & Escala likert 1-5 & Pettigrew et al., 2000 \\
\hline ORGANISATIONAL & $\begin{array}{l}\text { ¿Cuántos niveles or- } \\
\text { ganizativos hay entre } \\
\text { LEVELS (FLAT) } \\
\text { el directivo con el ni- } \\
\text { vel de responsabilidad } \\
\text { más bajo y el gerente } \\
\text { más elevado? (Por fa- } \\
\text { vor cuente la linea más } \\
\text { larga) }\end{array}$ & \\
\hline
\end{tabular}

FUENTE: Elaboración propia a partir PETTIGREW et al. (2000) y WHITTINGTON et al. (1999).

\section{- Descentralización}

Esta variable hace referencia al lugar o nivel en el que se toman las decisiones en la empresa. La escala utilizada en este trabajo ha sido adaptada de la escala de descentralización operativa presentada por Baum y Wally (2003), que realizaron su propuesta basándose en las apreciaciones del trabajo seminal de Khandwalla (1977).

\section{CUADRO 2}

\section{ESCALA DESCENTRALIZACIÓN OPERATIVA}

\begin{tabular}{|l|l|l|}
\hline Grado de acuerdo con las siguientes afirmaciones: \\
\hline DEC_Op1 & $\begin{array}{l}\text { Los trabajadores tienen libertad para tomar decisiones } \\
\text { operativas sobre problemas relacionados con el servicio y } \\
\text { los clientes }\end{array}$ & Escala Tipo likert 1-5 \\
\cline { 1 - 2 } DEC_Op2_inv & $\begin{array}{l}\text { Las personas involucradas en la planificación estratégica } \\
\text { toman diariamente decisiones relacionadas con las } \\
\text { operaciones de línea (INVERSA) }\end{array}$ & \\
\cline { 1 - 2 } DEC_Op3 & $\begin{array}{l}\text { Nuestros trabajadores de línea dirían que tienen libertad } \\
\text { para cambiar las cosas con el fin de mejorar el servicio }\end{array}$ & \\
\hline
\end{tabular}

FUENTE: Elaboración propia a partir de BAUM y WALLY (2003). 


\section{- Formalización}

La formalización es, del mismo modo, una variable clásica en la medición de las características de la estructura (Pugh et al., 1968; Khandwalla, 1977) Siguiendo el mismo esquema que en la variable anterior, en el caso de la formalización, se ha optado por la escala que proponen Baum y Wally (2003). En este caso, se ha considerado el especial interés que para la gestión del flujo de recursos tiene la distinción entre tareas rutinarias y tareas no rutinarias.

\section{CUADRO 3}

\section{ESCALA FORMALIZACIÓN}

\begin{tabular}{|l|l|l|}
\hline Formalización & \multicolumn{2}{|l|}{$\begin{array}{l}\text { Considere la diferencia entre tareas rutinarias y no-rutinarias en } \\
\text { su compañía. Las tareas rutinarias ocurren repetitivamente y las } \\
\text { no-rutinarias son inusuales. }\end{array}$} \\
\hline $\begin{array}{l}\text { Formalización de las tareas } \\
\text { rutinarias } \\
\text { (FORM_R) }\end{array}$ & Form_R1 & $\begin{array}{l}\text { Nuestra compañía tiene unos canales de comunica- } \\
\text { ción altamente formalizados para procesos y prác- } \\
\text { ticas rutinarias }\end{array}$ \\
\hline & Form_R2 & $\begin{array}{l}\text { Nuestro manual de procedimientos operativos nos } \\
\text { ayuda a hacer frente a los problemas rutinarios }\end{array}$ \\
\hline $\begin{array}{l}\text { Informalización de las tareas } \\
\text { rutinarias } \\
\text { (INFORM_NR) }\end{array}$ & INFORM_NR1 & $\begin{array}{l}\text { Puedo obtener la información que necesito cuando } \\
\text { me enfrento a problemas inusuales sin dirigirme a } \\
\text { otros canales. }\end{array}$ \\
\hline & INFORM_NR2 & $\begin{array}{l}\text { No hay instrucciones escritas para realizar tareas } \\
\text { no-rutinarias }\end{array}$ \\
\hline
\end{tabular}

FUENTE: Elaboración propia a partir de BAUM y WALLY (2003).

\subsubsection{Prácticas avanzadas de gestión ambiental}

Existen un gran número de taxonomías que describen la gestión ambiental en las empresas como un posicionamiento entre las posturas reactivas y proactivas (Aragón Correa, 1998). Por ejemplo, las prácticas de control de la polución o de cumplimiento, suelen incluir a prácticas «final de proceso» (end of pipe) que hacen que las organizaciones gestione sus desechos de forma responsable (Hart, 1995). Por otro lado, los enfoque de prevención de la contaminación, sistemáticamente aplican métodos proactivos basados en los principios de la gestión de la calidad total a su relación con el medio ambiente (Roome, 1992). Estas prácticas han sido clasificadas como prácticas orientadas a la planificación, a las operaciones y a la comunicación (González de Benito y González de Benito, 2006).

Con el fin de evaluar el grado de implementación de estas prácticas, se han tenido en cuenta tanto el enfoque reactivo como el proactivo. Por tanto, se han incluido finalmente tres indicadores para determinar si las empresas analizadas elaboran informes ambientales, disponen de algún tipo de certificación ambiental, y recogen 
información sobre su impacto en un conjunto de áreas de rendimiento ambiental propuesto por la International Air Transport Association (IATA) (Hooper y Greenall, 2002). Estas áreas son convergentes con las propuestas por la OCDE en su Directiva Ambiental de 2003. En este punto no se examina el nivel de rendimiento ambiental, sino si se recoge información sobre estas áreas o no.

Finalmente, para valorar el grado de implementación de tecnologías más eficientes, se incluyó como variable la edad media de los aviones de cada una de las compañías. Habitualmente, los nuevos aviones incorporan nuevas tecnologías que tienden a ser más amigables con el medio ambiente y más eficientes consumo de combustible. Debido al ciclo medio de vida de un avión y a los costes de estas tecnologías, estas suelen instalarse mayoritariamente en aviones nuevos.

El Cuadro 4 recoge modo de resumen las variables incluidas en el modelo.

\section{CUADRO 4}

ESCALA PRÁCTICAS AMBIENTALES

\begin{tabular}{|l|l|l|l|}
\hline \multicolumn{1}{|c|}{ Variable } & \multicolumn{1}{|c|}{ Descripción } & \multicolumn{1}{c|}{ Medición } & Tipo de práctica \\
\hline ENV_CERT & $\begin{array}{l}\text { ¿Dispone su organización de } \\
\text { certificación ambiental de al- } \\
\text { gún tipo? (por ejemplo, ISO } \\
\text { 14001, EMAS) }\end{array}$ & Dicotómica Sí-No & Planificación \\
\hline ENV_MESU & $\begin{array}{l}\text { ¿Recoge su organización in- } \\
\text { formación sobre el impacto } \\
\text { ambiental en alguna de las } \\
\text { siguientes áreas? }\end{array}$ & $\begin{array}{l}\sum \text { N. de áreas en el que la } \\
\text { empresa responde afirmativa- } \\
\text { mente }\end{array}$ & Planificación \\
\hline ENV_REPO & $\begin{array}{l}\text { ¿Elabora su organización al- } \\
\text { gún tipo de informe ambien- } \\
\text { tal? }\end{array}$ & Dicotómica Sí-No & Comunicación \\
\hline FLEET_MO & Edad media de la flota & & Operaciones \\
\hline
\end{tabular}

FUENTE: Elaboración propia.

\subsubsection{Variable de control (SIZE)}

El tamaño de la empresa fue incluido como una variable de control en el modelo final. Se estableció como el número medio de trabajadores de la empresa en los años 2007, 2008 y 2009.

\subsection{Metodología y resultados}

El análisis de datos fue realizado usando mínimos cuadrados parciales (Partial Least Squares, PLS), una técnica de modelización basada en ecuaciones estructurales que usa un sistema de estimación basado en componentes principales (Chin, 1998). 
Para comprobar las hipótesis, PLS ofrece diversas alternativas, aunque Chin (1998) recomienda el procedimiento denominado «bootstrapping». Basándonos en el trabajo de Barclay et al. (1995), el trabajo fue analizado e interpretado secuencialmente en dos etapas: (1) evaluación de la fiabilidad y validez del modelo de medida y (2) evaluación del modelo estructural.

\subsubsection{Modelo de medida}

La práctica común recomienda la evaluación del modelo de media examinando las correlaciones entre constructos, las correlaciones entre los constructos y los ítems, las alfas de Cronbach de las diferentes escalas, así como la fiabilidad compuesta y la varianza media extraída (Average Variance Extracted, AVE) para cada constructo (Cepeda, 2006). En PLS, los indicadores reflectivos son determinados por el constructo, y por tanto covarían al nivel de dicho constructo (Hulland, 1999). En nuestro modelo, todas las escalas utilizadas hacen uso de ítems reflectivos.

La fiabilidad individual de los ítems es evaluada en PLS examinando las cargas de las medidas sobre su respectivo constructo. En el modelo final, se han mantenido todos los ítems iniciales debido a que la potencial pérdida de información derivada de su eliminación un justificaba la mejora del modelo en términos de fiabilidad.

La fiabilidad del constructo ha sido evaluada usando dos medidas de consistencia interna, el alfa de Cronbach y la fiabilidad compuesta. La interpretación de ambos valores es similar, aunque la fiabilidad compuesta es una media más afinada en tanto en cuanto que no asume un reparto de los pesos de los items igualitario (Barclay et al., 1995). Nunnally (1978) sugiere 0,70 como un nivel de comparación base para una fiabilidad «modesta», aplicable especialmente en las etapas iniciales del desarrollo de la investigación, y un 0,80 como un nivel de fiabilidad más «estricto», aplicable a la investigación básica. Tal y como se muestra en el Cuadro 5, si bien el alfa de Cronbach no llega en algunos casos al límite establecido, la fiabilidad compuesta si que excede el nivel de 0,7 en todos los casos.

La validez discriminante representa el extremo hasta el cual las medidas de un constructo determinado son diferentes de otros constructos presentes en el mismo modelo. La validez discriminante ha sido evaluada de dos formas distintas (Chin, 1998). En primer lugar se ha comparado la raíz cuadrada del AVE (mostrada en la diagonal en el Cuadro 5) con las correlaciones entre los distintos constructos (representada por las figuras en las intersecciones entre las variables. Dado que la AVE es un indicador de la varianza capturada por el constructo en relación a la varianza total del erro de medida, sus valores deben exceder el nivel de 0,5 (Barclay et al., 1995). El Cuadro 5 muestra que la raíz cuadrada de AVE es mayor que la correlación entre los constructos, lo que sugiere que, en promedio, cada constructo se relaciona con mayor fortaleza con sus medidas que con las otras presentes en el modelo. 
CUADRO 5

INTERCORRELACIONES Y CONSISTENCIA INTERNA DE LOS CONSTRUCTOS

\begin{tabular}{|l|cc|ccccc|}
\hline \multicolumn{1}{|c|}{$\begin{array}{c}\text { Cons- } \\
\text { tructos }\end{array}$} & $\begin{array}{l}\text { Fiabilidad } \\
\text { compuesta }\end{array}$ & $\begin{array}{c}\text { Alfa de } \\
\text { Cronbach }\end{array}$ & $\begin{array}{c}\text { ENV_- } \\
\text { RESP }\end{array}$ & $\begin{array}{c}\text { FORM_ } \\
\text { RUT }\end{array}$ & $\begin{array}{c}\text { INFORM_ } \\
\text { NON_ } \\
\text { RUT }\end{array}$ & HORIZ & DEC \\
\hline $\begin{array}{l}\text { Prácticas am- } \\
\text { bientales (ENV_- } \\
\text { RESP) }\end{array}$ & 0,847 & 0,761 & $\mathbf{0 , 7 6 2}$ & & & \\
\hline $\begin{array}{l}\text { Formalización de } \\
\text { tareas rutinarias } \\
\text { (FORM_RUT) }\end{array}$ & 0,861 & 0,686 & 0,199 & $\mathbf{0 , 8 7 0}$ & & \\
\hline $\begin{array}{l}\text { Informalización } \\
\text { de tareas no } \\
\text { rutinarias (IN- } \\
\begin{array}{l}\text { FORM_NON_ } \\
\text { RUT) }\end{array}\end{array}$ & 0,844 & 0,685 & 0,184 & $-0,222$ & $\mathbf{0 , 8 5 6}$ & \\
\hline $\begin{array}{l}\text { Horizontalidad } \\
\text { (HORIZ) }\end{array}$ & 0,858 & 0,769 & 0,392 & 0,119 & 0,279 & $\mathbf{0 , 7 4 8}$ & \\
\hline $\begin{array}{l}\text { Decentralización } \\
\text { (DEC) }\end{array}$ & 0,745 & 0,546 & 0,372 & 0,088 & 0,414 & 0,369 & $\mathbf{0 , 7 0 6}$ \\
\hline
\end{tabular}

FUENTE: Elaboración propia.

El segundo procedimiento usado para evaluar la validez discriminante consiste en evaluar cómo se relaciona cada ítem con los constructos presentes en el modelo. Con este fin, hemos examinado las cargas de los ítems sobre su constructo y en el resto de constructos del modelo (cargas cruzadas). Todas las cargas de los ítems mostraron ser mayores en su propio constructo que en los otros. Además, todos los constructos comparten más varianza con su constructo que con los otros, por lo que podemos concluir que, estos resultados, colectivamente, dan soporte a la validez convergente y discriminante de las escalas utilizadas.

\subsubsection{Modelo estructural}

El objetivo principal de PLS es la minimización del error, o equivalentemente maximizar la varianza explicada, en todos los constructos endógenos. El grado en el que un modelo particular de PLS satisface este objetivo puede determinarse examinando los valores del R2 de los constructos dependientes (endógenos). Adicionalmente, las relaciones entre las variables pueden evaluarse a través de los valores de los coeficientes y su significatividad estadística.

El Gráfico 1 muestra el modelo estructural evaluado. Los valores incluidos dentro de las circunferencias que representan las variables endógenas muestran los valores del R2. 


\section{GRÁFICO 1}

MODELO ESTRUCTURAL

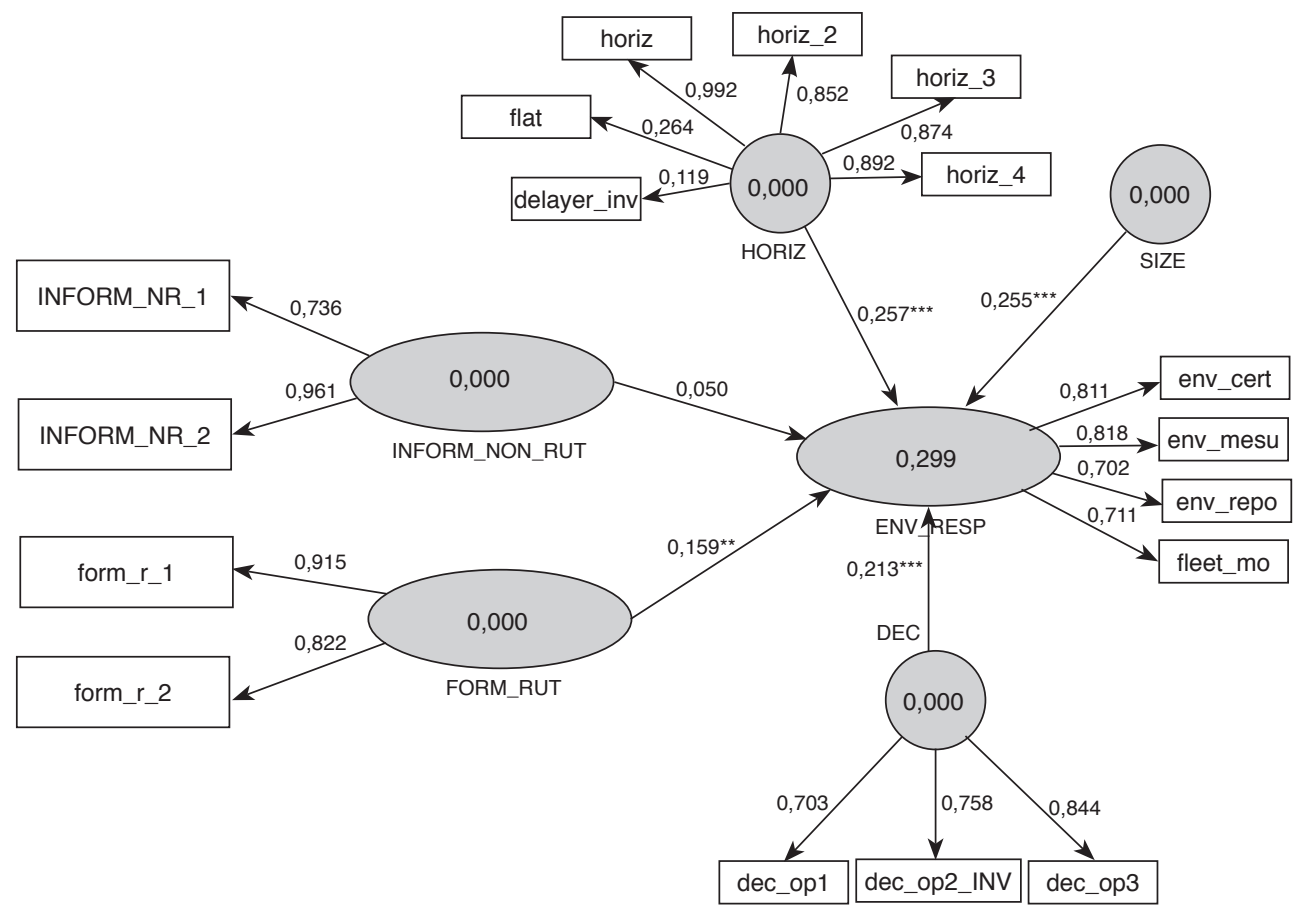

NOTA: $* * \mathrm{p}<0,01 ; * * * \mathrm{p}<0,001$ (distribución t-Student de dos colas con 499 grados de libertad).

Los resultados obtenidos sugieren la suficiencia del poder predictivo de este modelo. El valor del R2 de la variable dependiente excede el nivel de corte de 0.1 propuesto por Falk y Miller (1992).

Wold (1982) propuso el uso general del test de Stone-Geisser para la evaluación de la relevancia predictiva de un constructo. La aplicación de este test sigue un proceso de «blindfolding» que posibilita la construcción del indicador Q2 (1-SSE/SSO). La interpretación de este valor toma el 0 como nivel de referencia. De esta forma, se dice que el modelo tiene valor predictivo cuando el indicador es positivo (Sellin, 1995).

Los resultados del análisis del modelo muestran un valor del indicador Q2 para la variable endógena del modelo del 0,151 , lo que apunta a la viabilidad predictora del modelo propuesto.

La segunda parte del comentario sobre la evaluación del modelo estructural analiza la intensidad de la predicción de la variable endógena por las variables exógenas. Evaluar la dirección y significatividad de las relaciones modelizadas nos permite contrastar las hipótesis propuestas. 
La Hipótesis 1 sugería una relación directa y positiva entre el desarrollo de una estructuras más horizontales y con menor número de niveles jerárquicos. Los resultados del presente estudio dan soporte a esta hipótesis.

La Hipótesis 2 planteaba que un mayor nivel de descentralización en las operaciones está relacionado directa y positivamente con el desarrollo de prácticas avanzadas de gestión ambiental. Los resultados obtenidos apoyan esta proposición.

La Hipótesis 3 se estructuró en dos sub-hipótesis que han obtenido resultados contradictorios. Se distinguía entre tareas rutinarias y no rutinarias, proponiendo que la formalización sería útil en las primeras, pero no para las segundas. Los resultados del análisis realizado muestran que esta relación solo es significativa en el caso de la formalización de las tareas rutinarias. La propuesta que planteaba un impacto positivo de la informalización de las tareas menos rutinarias no ha encontrado base en los datos estudiados.

\section{Discusión de los resultados y conclusiones}

En este artículo se han estudiado los antecedentes estructurales de las prácticas de gestión ambiental. Partiendo de una concepción de las prácticas ambientales en la que se incorporan prácticas de planificación operativas y de comunicación (González de Benito y González de Benito, 2006) se ha evaluado el impacto que tienen sobre ellas diversas características estructurales.

Las formas organizativas tradicionales, fundamentadas en los postulados burocráticos de formalización, centralización y verticalidad presentan ciertas rigideces que inhiben la creación de conocimiento de forma continuada (Child y McGrath, 2001). Para sortear estas dificultades, las empresas están desarrollando aproximaciones más orgánicas en las que se favorece la comunicación y la adaptación a distintas situaciones (Pettigrew et. al., 2003). En este artículo se ha evaluado cómo el cambio en la aplicación de estos principios del diseño organizativo hacia modelos menos formalizados, y más aplanados y descentralizados incide sobre las prácticas ambientales.

Estas prácticas suelen conllevar una revisión continua de muchos de los procesos internos con ánimo de mejora y están muy relacionadas con la innovación (Aragón Correa, 1998). La estructura existente en la organización se constituye por tanto como la base sobre la que se construyen los procesos que incorporan el conocimiento de los trabajadores y les permiten ser partícipes de las actividades de la organización en el plano ambiental. (Boiral, 2002; Russo y Harrison, 2005; Martínez del Río et al., 2012). Este trabajo contribuye a la extensión de esta corriente, mostrando cómo las estructuras que dan más responsabilidad a los trabajadores en la toma de decisiones y que favorecen la cercanía entre directivos y trabajadores (eliminando niveles jerárquicos intermedios) y favoreciendo la comunicación y la cooperación horizontal en la empresa ayudan a la implantación de estas prácticas ambientales. 
La implantación del conjunto de estas prácticas ambientales ha sido considerada como una capacidad dinámica (Aragón Correa, 1998). Estas capacidades tienen sus fundamentos en las prácticas, rutinas y estructuras de las organizaciones (Teece, 2007). Con el presente trabajo se ofrece un respaldo empírico a esta relación, lo que constituye una aportación en sí misma en un ámbito de estudio en el que predominan los estudios cualitativos.

Los resultados de este estudio dan soporte a estos planteamientos excepto en el caso de la formalización de prácticas de comunicación. En este que mejor ajustan con las prácticas ambientales son aquellas que favorecen la eficiencia y la estandarización en las tareas rutinarias. Se esperaba que en las tareas no rutinarias, dado que la descentralización de la toma de decisiones y el aplanamiento de la estructura tienden a capacitar al trabajador para que se implique en la actividad empresarial, la no formalización fuese más relevante (Boiral, 2002), pero no ha sido así. Tal vez esto se deba a que la mayor parte de las actividades ambientales sean de carácter rutinario o que los EMS recogen manuales de procedimientos en caso de eventualidades que reducen el número de ocasiones no rutinarias.

La sustitución del control directo que supone la eliminación de capas en la estructura de la empresa, requiere de la aplicación de mecanismos complementarios de coordinación. La formalización da seguridad al trabajador sobre lo que debe hacer y lo que se espera de él.

Es necesario valorar las peculiaridades del sector analizado con el fin de poder establecer implicaciones para la gestión. Las mejoras ambientales en la industria de la aviación están íntimamente ligadas con el desarrollo de nuevas tecnologías más ecoeficientes y de prácticas de gestión que pueden mejorar la eficiencia en las operaciones. En esta industria la eficiencia operativa y ambiental están altamente correlacionadas dado que un menor consumo de combustible implica menos emisiones de $\mathrm{CO} 2$. En la industria de la aviación, el impacto ambiental es contingente a un gran número de variables, tanto internas como externas. Por ejemplo, las emisiones de $\mathrm{CO} 2$ dependen obviamente de las características del avión empleado y de la tecnología que este incorpora, pero igualmente dependen del peso transportado por el avión, la ruta seguida o las condiciones meteorológicas que puedan afectar cada vuelo. De esta forma, es realmente complicado cuantificar exactamente las emisiones de cada compañía. Por tanto, las prácticas avanzadas de gestión ambiental en esta industria requieren un monitoreo regular de diversas medidas de impacto ambiental. La formalización de las tareas más rutinarias, unida a una mayor horizontalidad, pueden ayudar a establecer flujos de comunicación necesarios y ágiles para su medición continuada.

En la industria de la aviación, el tamaño medio de las compañías es relativamente elevado. Esto puede suponer ciertas dificultades en la comunicación interna. La implementación de prácticas ambientales requiere de coordinación entre los diferentes niveles de la organización (Russo y Fouts, 1997).

Con el fin de resolver estos problemas las empresas que ponen en marcha prácticas ambientales, hacen públicos sus esfuerzos a través de informes periódicos con 
el fin de alinear sus esfuerzos con los de diferentes grupos de interés Adicionalmente, investigaciones previas han mostrado que existe una relación positiva entre la incorporación de prácticas y estrategias ambientales y la redacción de informes de sostenibilidad (Mio, 2010). En este sentido, las características de la estructura que favorecen la coordinación entre diversas unidades se revelan especialmente útiles. Así, la implementación de estructuras con un menor número de niveles jerárquicos y el uso de equipos multifuncionales puede ayudar a coordinar tareas diversas a la vez que permite que la información se traslade de forma eficiente de una parte a otra de la organización. Igualmente, el registro sistemático y rutinario de información facilita la recopilación de datos relevantes para la elaboración de los informes de sostenibilidad.

Las implicaciones y conclusiones expuestas anteriormente, deben ser interpretadas con precaución teniendo en cuenta las limitaciones que puede presentar este trabajo y que pueden servir de punto de partida para trabajos posteriores. Así por ejemplo, la muestra empleada, aunque de tamaño suficiente para la metodología de análisis planteada, puede ofrecer resultados más nítidos con un tamaño muestral mayor. De igual forma, el hecho de que todas las respuestas provengan de un único respondente por empresa y que estas provengan de un único sector, puede introducir algunos sesgos en las respuestas que deben ser tenidos en cuenta a la hora de generalizar los resultados.

\section{Referencias bibliográficas}

[1] ADLER, N. y GELLMAN, A. (2012): «Strategies for managing risk in a changing aviation environment», Journal of Air Transport Management, 21, pp. 24-35.

[2] ADLER, P. S.; GOLDOFTAS, B. y LEVINE, D. I. (1999): «Flexibility versus efficiency? A case study of model changeovers in the Toyota production system», Organization Science, 10(1), pp. 43-68.

[3] AIKEN, M. y HAGE, U. (1971): «The organic organization and innovation», Sociology, 5, pp. 63-82.

[4] ARAGON CORREA, J. A. (1998): «Strategic proactivity and firm approach to the natural environment», Academy of Management Journal, 41(5), pp. 556-568.

[5] ARAGON CORREA, J. A. y SHARMA, S. (2003): «A contingent resource-based view of proactive corporate environmental strategy», The Academy of Management Review, 28(1), pp. 71-88.

[6] BARCLAY, D. W.; HIGGINS, C. y THOMPSON R. (1995): «The partial least squares (PLS) approach to causal modeling: Personal computer adaptation and use as an illustration», Technology Studies, 2(2), pp. 285-309.

[7] BAUM, J. R. y WALLY, S. (2003): «Strategic decision speed and firm performance», Strategic Management Journal, 24(11), pp. 1107-1130.

[8] BOIRAL, O. (2002): «Tacit knowledge and environmental management», Long Range Planning, 35, pp. 291-317.

[9] BOIRAL, O. (2007): «Corporate greening through ISO 14001: a rational myth?», Organization Science, 18, pp. 127-146. 
[10] CEPEDA, G. (2006): «Understanding the link between knowledge management and firm performance: articulating and codifying critical knowledge areas», International Journal of Knowledge and Learning, 2, pp. 238-262.

[11] CHILD, J. (1974): «What Determines Organization Performance - Universals vs It-AllDepends», Organizational Dynamics, 3(1), pp. 2-18.

[12] CHILD, J. y MCGRATH, R. G. (2001): «Organizations unfettered: Organizational form in an information-intensive economy», Academy of Management Journal, 44(6), pp. 1135-1148.

[13] CHIN, W. W. (1998): «The partial least squares approach for structural equation modeling», en Marcoulides, G. A. (ed.), Modern Methods for Business Research, Lawrence Erlbaum Associates: Mahwah, NJ.

[14] DEL BRÍO, J.A. y JUNQUERA, B. (2003): «A review of the literature on environmental innovation management in SMEs: Implications for public policies», Technovation, 23, pp. 939-948.

[15] DEL BRÍO, J. A.; FERNÁNDEZ, E. y JUNQUERA, B. (2007): «Management and employee involvement in achieving an environmental action-based competitive advantage: an empirical study», The International Journal of Human Resource Management, 18(4), pp. 491-522.

[16] FALK, R. F. y MILLER, N. B. (1992): A primer for soft modelling (1st ed.), University of Akron Press, Akron, Ohio.

[17] FORD, J. D. y SLOCUM, J. W. jr. (1977): «Size, Technology, Environment and the Structure of Organizations», Academy of Management Review, 2(4), pp. 561-575.

[18] FORNELL, C.; LORANGE, P. y ROOS, J. (1990): «The cooperative venture formation process: A latent variable structural modeling approach», Management Science, 36(10), pp. 1246-1255.

[19] FREEMAN, S. J. y CAMERON, K. S. (1993): «Organizational Downsizing, a Convergence and Reorientation Framework», Organization Science, 4(1), pp. 10-29.

[20] GALUNIC, D. C. y EISENHARDT, K. M. (2001): «Architectural innovation and modular corporate forms», Academy of Management Journal, 44(6), pp. 1229-1249.

[21] GONZÁLEZ BENITO, J. y GONZÁLEZ BENITO, O. (2006): «A review of determinant factors of environmental proactivity», Business Strategy and the Environment, 15, pp. 87-102.

[22] GREENWOOD, R. y MILLER, D. (2010): «Tackling design anew: getting back to the heart of organization theory», Academy of Management Perspectives, noviembre, pp. 78-88.

[23] HAGE, J. y AIKEN, M. (1967): «Relationship of Centralization to Other Structural Properties», Administrative Science Quarterly, 12(1), pp. 72-92.

[24] HAGE, J. y AIKEN, M. (1969): «Routine Technology, Social Structure, and Organizational Goals», Administrative Science Quarterly, 14, pp. 366-376.

[25] HAMBRICK, R. C.; GELETKANYCZ, M. A. y FREDRICKSON, J. W. (1993): «Top executive commitment to the status quo: Some tests of its determinants», Strategic Management Journal, 14(6), pp. 401-418.

[26] HART, S. L. (1995): «A natural-resource-based view of the firm», Academy of Management Review, 20, pp. 986-1014.

[27] HOOPER, P. D. y GREENALL, A. (2002): «Exploring the potential for environmental performance benchmarking in the airline sector», Benchmarking: An International Journal, 12(2), pp. 151-165. 
[28] HULLAND, J. (1999): «Use of partial least squares (PLS) in strategic management research. A review of four recent studies», Strategic Management Journal, 20(2), pp. 195.

[29] KHANDWALLA, P. N. (1977): The design of organizations. Ed. Harcourt Brace Jovanovich, New York.

[30] KITAZAWA, S. y SARKIS, J. (2000): «The relationship between ISO 14001 and continuous source reduction programs», International Journal of Operations and Production Management, 20 (2), pp. 225-248.

[31] LENOX, M. y KING, A. (2004): «Prospects for developing absorptive capacity through internal information provision», Strategic Management Journal, 25(4), pp. 331-345.

[32] MARTÍNEZ DEL RÍO, J.; CÉSPEDES LORENTE, J. y CARMONA MORENO, E. (2012): «High-involvement work practices and environmental capabilities: how HIWPS create environmentally based sustainable competitive advantages», Human Resource Management, 51(6), pp. 827-850

[33] MINTZBERG,H. (1979): The structuring of organizations: a synthesis of the research, Ed. Prentice-Hall, Englewood Cliffs, N.J.

[34] MIO, C. (2010): «Corporate social reporting in Italian multi-utility companies: an empirical analysis», Corporate Social Responsibility and Environmental Management, 17, pp. 247-271.

[35] NUNNALLY, J. (1978): Psychometric Theory, 2. ${ }^{a}$ ed., McGraw-Hill, New York.

[36] PALMER, I.; BENVENISTE, J. y DUNFORD, R. (2007): «New Organizational Forms: Towards a Generative Dialogue», Organization Studies, 28(12), pp. 1829-1847.

[37] PETTIGREW, A. (ed.). (2003): Innovative Forms of Organizing: International Perspectives, Thousand Oaks: Sage Publications, London.

[38] PUGH, D. S.; HICKSON, D. I. y TURNER, C. (1968): «Dimensions of Organization Structure», Administrative Science Quarterly, 13, pp. 65-105.

[39] QUINN, J. B. (1985): «Managing Innovation: Controlled Chaos», Harvard Business Review, 63(3), pp. 73-84.

[40] ROOME, N. (1992): «Developing environmental management strategies», Business Strategy and the Environment, 1(1), pp. 11-24.

[41] RUSSO, M. V. y FOUTS, P. A. (1997): «A resource-based perspective on corporate environmental performance and profitability», Academy of Management Journal, 40(4), pp. 534-559.

[42] RUSSO, M. V. y HARRISON, N.S. (2005): «Organizational design and environmental performance: Clues from the electronics industry», Academy of Management Journal, 48(4), pp. 582-593.

[43] SANCHEZ BUENO, M. J. y SUAREZ GONZALEZ, I. (2010): «Towards new organizational forms», International Journal of Organizational Analysis, 18(3), pp. 340-357.

[44] SELLIN, N. (1995): «Partial least squares modeling in research on educational achievement», en Bos, W. y Lehmann, R. H. (eds.), Reflections on Educational Achievement, Waxmann, New York.

[45] SHARMA, S. y VREDENBURG, H. (1998): «Proactive corporate environmental strategy and the development of competitively valuable organizational capabilities», Strategic Management Journal, 19(8), pp. 729-743.

[46] SHARMA, S.; PABLO, A. L. y VREDENBURG, H. (1999): «Corporate environmental responsiveness strategies: The importance of issue interpretation and organizational context», Journal of Applied Behavioral Science, 35, pp. 87-108. 
[47] TEECE, D. J. (2007): «Explicating dynamic capabilities: the nature and microfoundations of (sustainable) enterprise performance», Strategic Management Journal, 28(13), pp. 1319-1350.

[48] TEECE, D. J.; PISANO, G. y SHUEN, A. (1997): «Dynamic capabilities and strategic management», Strategic Management Journal, 18(7), pp. 509-533.

[49] TOSI, H. L.; KATZ, J. P. y GOMEZ-MEJIA, L.R. (1997): «Disaggregating the agency contract: the effects of monitoring, incentive alignment, and term in office on agent decision making», Academy of Management Journal, 40(3), pp. 584-602.

[50] TUSHMAN, M. y ANDERSON, P. (1997): Managing strategic innovation and change: a collection of readings, Oxford University Press, New York.

[51] VAN DE VEN, A. H. (1976): «A framework for organization assessment», Academy of Management, The Academy of Management Review, 1(1), pp. 64-78.

[52] WADDOCK, S.A.; BODWELL, C. y GRAVES, S. R. (2003): «Responsibility: the new business imperative», Academy of Management Executive, 16(2), pp. 132-148.

[53] WOLD, H. (1982): «Systems Under Indirect Observation Using PLS», en Fornell, C. [ed.], A Second Generation of Multivariate Analysis, 1, pp. 325-347, Praeger Publishers, New York. 